\title{
Semiological Profile of Documented Apical Periodontitis: Case of the Clinic of Operative Dentistry and Endodontics (ODE) of the Department of Dentistry of Dakar
}

\author{
Ndiaye $\mathrm{D}^{*}$, Gnagne Koffi $\mathrm{Y}^{2}$, Sarr $\mathrm{M}^{1}$, Seck $\mathrm{A}^{1}$, Bane $\mathrm{K}^{1}$, Faye $\mathrm{B}^{1}$ and Machtou $\mathrm{P}^{3}$ \\ ${ }^{1}$ Department of Conservative Dentistry, Department of Odontology, Faculty of Medicine- Cheikh Anta Diop Uni- \\ versity of Dakar, Senegal \\ ${ }^{2}$ Department of Conservative Dentistry and Endodontics, Faculty of Odontology-Houphouet Boigny University of \\ Abidjan, Ivory Coast \\ ${ }^{3}$ Pitie Salpêtrière University Hospital of Paris, France
}

*Corresponding author: Ndiaye D, Department of Conservative Dentistry, Department of Odontology, Faculty of Medicine- Cheikh Anta Diop University of Dakar, Senegal, Tel : +221776150167 E-mail: juma.@ yahoo.fr

Citation: Ndiaye D, Gnagne Koffi Y, Sarr M, Seck A, Bane K, et al. (2016) Semiological Profile of Documented Apical Periodontitis: Case of the Clinic of Operative Dentistry and Endodontics (ODE) of the Department of Dentistry of Dakar. J Dent Oral Care Med 2(3): 305. doi: 10.15744/2454-3276.2.305

Received Date: May 09, 2016 Accepted Date: August 30, 2016 Published Date: August 31, 2016

\begin{abstract}
Objectives: The prevalence of teeth carriers of periodontitis apical is universally high. Only the decontamination of the root canal system through cleaning, it's shaping and its waterproof filling can ensure the eradication of infection at the level of the peri apex. The purpose of this prospective study was to establish the semiological profile of different types of periodontitis apical. The different characteristics used are subjective signs reported by the patient about the pain but also the clinical and radiographic features of each tooth with apical periodontitis.

Material and Methods: The data were collected with an investigation form to identify the patient and meet the different clinical elements. All patients presenting to the clinic of ODE and having one or more teeth with the diagnosis of apical periodontitis was made were included in the study. A survey sheet crafted based on the modified Stephan model with the following headings namely: identification of the patient, the elements intra and extra oral, different etiologies, clinical and radiological diagnosis, recommended treatments.

The software Sphin ${ }^{2}$ Copyright 1986-2003 has been used for data input and analysis. The test of chi2 was used to study the association between the predefined variables.

Results: One hundred and six (106) teeth were diagnosed among 85 patients. The predominants signs were the absence of pain and the presence of apical radio translucency associated with swelling. In $60.3 \%$ of the cases the etiology was caries with pain during consultation in $56.6 \%$ of cases. The maxillary sector was the most affected $(39.6 \%)$ and the apical periodontitis chronic was the type of pathology the most met (40.5\%).

Conclusion: This retrospective study will enable practitioners to have a better diagnostic approach and therapeutic of apical periodontitis.

Keywords: Apical periodontitis; Semiological profile; Diagnostic criteria
\end{abstract}

\section{Clinical Significance}

This study will allow practitioners to dispose of diagnostic criteria and provide the most appropriate therapeutics to each type of apical periodontitis encountered.

\section{Introduction}

The apical periodontitis are of inflammatory lesions of the deep periradicular periodontitis, mainly in the periapical region consecutive to the bacterial infection of the endodontitis $[1,2]$. In fact, an untreated, sick pulp evolved quietly to the necrosis. The bacteria that colonize as well the system endodontic treatments produce toxins which, little by little drain in the periapex through principal channels and side [3]. Several studies on the epidemiology of these lesions have been carried out [4,5]. It shows that the prevalence of 
teeth carriers of periodontitis apical is universally high and varies between 15 and 75\% but largely under estimated in many countries [5]. Because of their multiple clinical forms, they have been the subject of several classifications with the last being that of American Association of Endodontists (Normal Apical Tissues, Symptomatic Apical Periodontitis, Asymptomatic Apical Periodontitis, Chronic Apical Abscess, Acute Apical Abscess, Condensing Osteitis) [2]. The patients are unaware of them in their chronic and asymptomatic phases hence the interest, for the practitioner, to correctly diagnose these pathologies and etablish a good treatment plan.

Few studies have focused on the semiological profile of these documented periodontitis apical [4]. It is in this perspective that this work has been carried out with the main objective to establish the semiological profile of different types of periodontitis apical encountered in a Senegalese population on the base of the characteristics of the pain but also clinical and radiological signs of each tooth having a apical periodontitis. It will allow practitioners to dispose of diagnostic criteria and perform the most appropriate therapeutics to each type of apical periodontitis encountered.

\section{Material and Methods}

It is a prospective study conducted during the academic year $2014-2015$ (October 07, 2014 to $31^{\text {st }}$ of July 2015). The study population was composed of patient's consultant at the clinic of Operative Dentistry and Endodontics of the department of Dentistry at the University Cheikh - Anta Diop in Dakar. Have been included in the study all patients who have given their consent, with one or several teeth diagnosed with apical periodontitis during the clinical examination. The data have been collected with a questionnaire which allowed identifying the patient and meeting the different clinical elements.

The hardware of clinical examination was composed of a plateau of examination, of Pharmaethyl $1^{\oplus}$, periodontal probe type WHO, movies retro-alveolar, film holders (HAWE Super Bite, Endo Ray II), of a x-ray device of $70 \mathrm{KV}$, films for the radiographs dental panoramic, pliers film door, a light box, a magnifying glass and a digital camera.

To be able to establish the semiological profile of the apical periodontitis, a clinical examination of each patient was carried out supplemented by radiography.

The clinical examination included an interrogation, an inspection, a palpation, a cold test, percussion and a poll periodontal charting. A radiography retro orthocentred alveolar or offset was carried out according to the technique of parallel plans at the level of each causal tooth. For the case of apical lesions of large extent, a panoramic radiography was performed to assess the report of the periapical lesion with the teeth and neighboring organs.

The software Sphinx ${ }^{2}$ Copyright 1986-2003 has been used for data input and analysis. The test of chi2 has allowed us to study the association between the predefined variables. The threshold of significance was set at $\mathrm{p}<0.05$.

\section{Results}

It has been recorded 106 teeth with apical periodontitis among 85 patients. The quarter (25.76\%) of patients had at least one tooth affected with apical periodontitis. Males accounted for $58.8 \%$ of the population being studied and $41.2 \%(n=35)$ were women. The distribution between the two groups is not statistically significant $(p=0.08)$. The average age of patients is $29.13 \pm 10.59$ years with a minimum of 17 years and a maximum of 77 years. From a socio-economic stand point the students constituted the prevalent social class (41.2\%). The distribution of the study population according to the state of health has shown that $66.3 \%$ had a good general condition, $7.9 \%$ had a gastroduodenal ulcer, $6.7 \%$ were asthmatic, and $5.6 \%$ had a low blood pressure $4.5 \%$ was affected by a sickle cell anemia, $2.2 \%$ by diabetes and $6.7 \%$ by other pathologies. The 106 cases of documented periodontitis apical discovered in consultation had as chief complaint the pain in $56.6 \%$ of cases. $30.2 \%$ of the cases were discovered by chance and $13.2 \%$ were referred by other services (oral surgery, periodontology and prosthodontic). More than half of the patients (51.8\%) had an oral dental hygiene defective with soft deposits and tartar generalized, $37.6 \%$ had an average hygiene and $10.6 \%$ a good hygiene. The difference is very significant $(\mathrm{p}=0.00)$. In the most cases, the patient set of teeth of was incomplete $(68.2 \%)$. The difference between the two set of teeth was statistically significant $(\mathrm{p}=0.00)$. The groups of teeth affected by apical periodontitis are mainly: the mandibular incisors (28 central incisors and 10 lateral incisors), followed by the mandibular molars (14 first molars and 14 second molars). The distribution between the different sectors is very significant $(\mathrm{p}=0.00)$. The dominating etiology was the dental caries $(60.3 \%)$, followed by the trauma (31.2\%) and an endodontic treatment failure (8.8\%). The asymptomatic apical periodontitis is the most frequent $40.6 \%$, followed by the chronic apical abscess (17\%) and the symptomatic apical periodontitis $(17 \%)$, and then comes the acute apical abscess (8.5\%). The difference between the different type of pathology is very significant (p $=0.00$ ). The distribution of type of apical periodontitis according to the location of the teeth showed that the asymptomatic apical periodontitis with fistula occurs mainly at the level of maxillary anterior teeth sector whereas for the posterior mandibular teeth sector; it is noted a predominance of apical periodontitis from the acute pathogenetic line (Figure1).

The study identified a semiological profile of different types of apical periodontitis diagnosed in order to establish their mapping (Table 1). It was noted in particular the scores in relation to the number of citations for each clinical sign and radiological. Thus the clinical signs, the complementary examinations and the radiological analyzes have led to the identification of diagnostic criteria major for the different pathologies of the peri apex. 


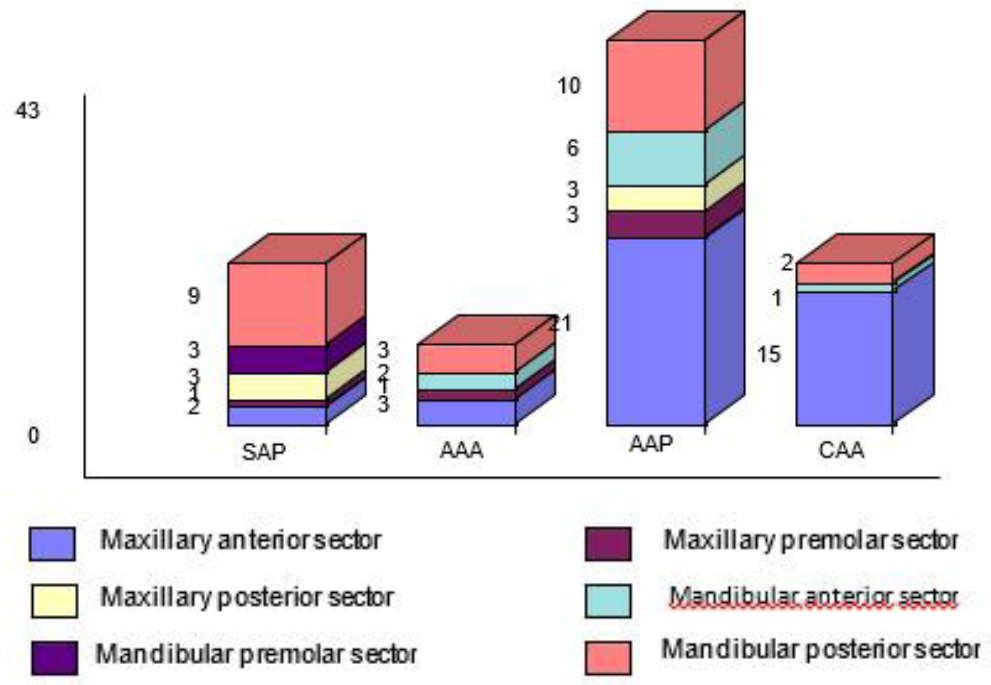

SAP = Symptomatic Apical Periodontitis; AAA = Acute Apical Abscess; $\mathrm{AAP}=$ Asymptomatic Apical Periodontitis; CAA = Chronic Apical Abscess

Figure 1: Type of apical periodontitis depending of the location of the teeth

Symptomatic Apical Periodontitis (SAP): The pain on percussion is one of the major signs, followed by pressure to the apical region. Sometimes there had spontaneous pain, the examples include pulp sensitivity to cold and pain to the percussion. These signs were encountered in $100 \%$ of cases and these characters were sometimes associated with pain on apical palpation (47.8\% of the cases). The thickened periodontal ligament is the main sign radiological (100\% of cases), it was followed by the presence of apical radiolucency $(27.3 \%$ of the cases) signing a bone lysis.

Acute Apical Abscess (AAC): clinical pathognomonic signs of the acute apical periodontitis with abscess were the presence of a swelling (100\%), the pain to percussion (100\%) and the absence of pulpal sensitivity (100\%). The pain to the apical palpation was noted in $88.9 \%$ of cases. The presence of an apical radiolucency, lysis of bone; is the major radiological sign ( $100 \%$ of cases). It was followed by the thickening periodontal ligament (88.9\%).

Asymptomatic Apical Periodontitis (AAP): the absence of pulpal sensitivity was the major clinical sign (100\% of cases) followed by the pain to percussion (93\%) and the tenderness to palpation apical (32.6\%). In $100 \%$ of cases, an apical radiolucency is observed, associated with a thickening periodontital ligament (86\%).

Chronic Apical Abscess (CAA): The presence of a fistula (100\% of cases) and the absence of the pulpal sensitivity (100\% of cases) were the clinical signs major. They were often associated with pain to apical palpation (44.4\%). The apical radiolucency and the thickening periodontal ligament appeared in all the cases.

\begin{tabular}{|c|c|c|}
\hline & Clinical signs & Radiological Signs \\
\hline $\begin{array}{l}\text { Symptomatic Apical } \\
\text { Periodontitis }\end{array}$ & $\begin{array}{c}\text { Spontaneous Pain }^{+++} \\
\text {Pain to percussion } \\
\text { Lack of pulpal sensitivity }^{+++} \\
\text {Pain on palpation apical }\end{array}$ & $\begin{array}{l}\text { Thickening periodontal } \\
\text { ligament } \\
\text { Radiolucency, image of } \\
\text { bone lysis }{ }^{+}\end{array}$ \\
\hline Acute Apical Abscess & $\begin{array}{c}\text { Swelling }{ }^{+++} \\
\text {Pain to percussion }{ }^{+} \\
\text {Lack of pulpal sensitivity }^{+++} \\
\text {Pain to apical palpation }{ }^{+}\end{array}$ & $\begin{array}{l}\text { Radiolucency, image of } \\
\text { bone lysis } \\
\text { Thickening periodontal } \\
\text { ligament }\end{array}$ \\
\hline Chronic Apical Abscess & $\begin{array}{l}\text { Presence of fistula }{ }^{+++} \\
\text {Lack of sensitivity } \\
\text { therefore } \\
\text { Pain to apical palpation }\end{array}$ & $\begin{array}{l}\text { Radiolucency, image of } \\
\text { bone lysis } \\
\text { Thickening periodontal } \\
\text { ligament }\end{array}$ \\
\hline
\end{tabular}

Table 1: The diagnostic criteria of the different types of apical periodontitis encountered

\section{Discussion}

Our study allowed establishing the diagnosis profile of different types of apical periodontitis encountered in the clinic of Operative Dentistry and Endodontics of the department of dentistry of Dakar.

\section{Patients}

Men predominate in general and represent $58.9 \%$ of the sample, but the difference is not statistically significant. The average age of patients is $29.13 \pm 10.59$ with a minimum of 17 years and a maximum of 77 years. These results are consistent with a study 
conducted in Nigeria who has shown that young people are more affected than the elderly [5]. The fact that the survey is carried out in academia also explains this predominance of young people. In fact, the students constitute the class majority in our sample $(41.2 \%)$; the clinic of O. D. E. is located within the university and the campus social only has an office.

The major part of the patients carrying apical periodontitis showed a good general condition (66.3\%), two patients with diabetes (Type II) balanced have been listed in our cohort and were normally supported while no cases of patient at risk (cardiopathe, uraemic patients, immuno etc.) has been noted. These data certainly show that the general condition does not affect at all on the prevalence of documented apical periodontitis. A recent study shows even their independence with the diseases of the coronary artery [6].

\section{Clinical Data}

Most of the 106 teeth with apical periodontitis (56.6\%) have been treated in emergency (consultation reason: pain) in adequacy with all the studies on the typology of consultants in endodontics. In fact, Arens et al. [8] in his study had found that the pain was more than $60 \%$ of the reasons for consultation in endodontics. Quiñonez, et al. [9] have also demonstrated that among the orofacial pain bringing patients to consult in emergency, $85 \%$ are related to endodontic treatments. The fortuitous discovery of apical asymptomatic periodontitis (30.2\%) in patients consulting for other reasons or for an aesthetic issue shows that these last do not cause patients to consult as highlighted by other authors [10].

Among the 85 patients examined, $44(51.8 \%)$ had a poor oral hygiene. This high percentage shows that the poor oral hygiene is a factor of exposure to the onset of dental caries, and therefore of the apical periodontitis as it has been noted in many studies [11].

The maxillary anterior sector is the most affected with $39.6 \%$ of apical periodontitis, monitoring of the mandibular posterior sector (28.3\% of cases). This can have a dual explanation: on the one hand, the maxillary anterior sector is the privileged place of the trauma on the other hand the mandibular posterior sector is the preferred location of caries [12]. These results are also confirmed by the work of Eriksen, et al. [13] who found that the apical periodontitis affects mainly the upper side incisors, the first upper molars and first lower molars.

The carious etiology dominated with a rate of $60.3 \%$ and confirmed the place that dental caries occupies in the oral pathology (4 e global scourge) and particularly in the mandibular posterior sector. The traumas represent $31.2 \%$ of the cases, this can be explained by the fact that the population is young and male majority, subject to trauma during sports or fights 10 to 15 years earlier [14]. The failure of undergone endodontic treatment as predisposing factor of the apical periodontitis is recognized through numerous studies [15-17]. Only $8.8 \%$ of the cases of documented apical periodontitis fell within failed endodontic treatments in our study, probably because of the difficult access to treatment and of the unfavourable socio-economic level. Buckley and Spangberg showed that $31.3 \%$ of the documented periodontitis apical are due to endodontic treatments poorly performed [18]. According to their results the probability for a patient to develop an apical periodontitis is 5 to 10 times higher for treated teeth than for pathological teeth for which no undergone endodontic treatment has been established. There is a strong correlation between the teeth remaining, the number of root canal teeth and the existence of apical lesions [13]; 68.2\% of our patients had an incomplete set of teeth for $90.6 \%$ of teeth apical lesion.

\section{Semiological profile of documented periodontitis apical}

Compared to the semiological profile proposed by the American Dental Association, it has been obtained confirmation from the spontaneous pain, of a positive response to the sensitivity tests, the pain to the percussion and widening periapical ligament space were the pathognomonic signs of the symptomatic apical periodontitis [2]. These signs were found in a few studies $[1,19]$. Therefore some authors didn't found clinical symptoms (no pain, no percussion or palpation) in the case of asymptomatic apical periodontitis. The signs of radiolucency and the thickening periodontal ligament were often encountered.

For the acute apical abscess, the cardinal signs are recovered: the pain to the contact, the thickening periodontal ligament, the painful apical palpation and the swelling.

The rarefaction bone in case of periapical osteosclerosis is confirmed in the case of chronic apical periodontitis. At the premolar sector level, this periapical ostesclerosis can make believe to a delayed healing in the controls in the medium and long term [20].

\section{Conclusion}

The apical periodontitis result from different attacks that can affect the pulp: dental caries, trauma, operative procedures, the periodontal disease, the absence of sealing of the endodontic fillings and coronal restorations that are associated with them.

There have been a variety of diagnostic classification systems advocated for determining endodontic disease. Unfortunately, the majority of them have been based upon histopathological findings rather than clinical findings, often leading to confusion, misleading terminology, and incorrect diagnoses. Therefore it is important to establish a proper diagnosis for a better management of patients with apical periodontitis. 


\section{Acknowledgment}

The authors wish to thank Prof. Pierre Machtou, Public Hospitals of Paris, Dentistry Service, Pitié Salpêtrière University Hospital, Paris, France, for his comments on early drafts of the manuscript.

\section{References}

1. Glickman GN (2009) AAE Consensus Conference on Diagnostic Terminology: background and perspectives. J Endod 35: 1619-20.

2. AAE Consensus Conference Recommended Diagnostic Terminology (2009). J Endod 35: 1634.

3. Tian Y, Zhang X, Zhang K, Song Z, Wang R, et al. (2013) Effect of Enterococcus faecalis lipoteichoic acid on apoptosis in human osteoblast-like cells. J Endod 39: $632-7$

4. Shahravan A, Haghdoost AA (2014) Endodontic epidemiology. Iran Endod J 9: 98-108.

5. LM Paes da Silva Ramos Fernandes, R Ordinola-Zapata, MA Húngaro Duarte, AL Alvares Capelozza (2013) Prevalence of apical periodontitis detected in cone beam CT images of a Brazilian subpopulation. Dentomaxillofac Radiol 42: 80179163.

6. Frisk F, Hugosson A, Kvist T (2015) Is apical periodontitis in root filled teeth associated with the type of restoration? Acta Odontol Scand 19: 1-7.

7. Costa TH, de Figueiredo Neto JA, de Oliveira AE, Lopes e Maia Mde F, de Almeida AL (2014) Association between chronic apical periodontitis and coronary artery disease. J Endod 40: 164-7.

8. Arens D, Gluskin AH, Peters CI, Peters OA (2009) Endodontic Emergencies and Their Treatment In: Practical Lessons in Endodontic Treatment. Chicago: Quintessence Publishing 271-6.

9. Quiñonez C, Gibson D, Jokovic A, Locker D (2009) Emergency department visits for dental care of nontraumatic origin. Community Dent Oral Epidemiol 37: 366-71.

10. Toure B, Faye B, Kane AW, Lo CM, Niang B, et al. (2011) Analysis of reasons for extraction of endodontically treated teeth: a prospective study. J Endod 37: $1512-5$.

11. Zhang S, Liu J, Lo EC, Chu CH (2013) Dental caries status of Dai preschool children in Yunnan Province, China. BMC Oral Health 13: 68.

12. Vashisth S, Bansal M, Gupta N (2014) Prevalence of traumatic injuries and knowledge regarding emergency care among 11-14 years government school children in rural area, Dehra, Kangra district, Himachal Pradesh. Oral Health Dent Manag 13: 666-8.

13. Eriksen HM (1991) Endodontology-epidemiologie considérations. Endod Dent Traumatol 7: 189-195.

14. Ramos-Jorge ML, Peres MA, Traebert J, Ghisi CZ, de Paiva SM, et al. (2008) Incidence of dental trauma among adolescents: a prospective cohort study. Dent Traumatol 24: 159-63.

15. Nur BG, Ok E, Altunsoy M, Ağlarci OS, Çolak M, et al. (2014) Evaluation of technical quality and periapical health of root-filled teeth by using cone-beam CT. J Appl Oral Sci 22: 502-8.

16. Khullar P, Raisingani D, Gupta S, Khatri RK (2013) A Survey Report on Effect of Root Canal Fillings and Coronal Restorations on the Periapical Status of Endodontically Treated Teeth in a Selected Group of Population. Int J Clin Pediatr Dent 6: 89-94.

17. Jersa I, Kundzina R (2013) Periapical status and quality of root fillings in a selected adult Riga population. Stomatologija 15: 73-7.

18. Buckley M, Spangberg LSW (1995) The prevalence and technical quality of endodontic treatment in an American subpopulation. Oral Surg oral Med oral pathol oral Radiol Endo 79: 92-100.

19. Gambiez A, Deveaux E (2006) Diagnosis in endodontics: pathologies. Real Clin 17: 291-306.

20. Sjögren U, Figdor D, Persson S, Sundqvist G (1997) Influence of infection at the time of root filling on the outcome of endodontic treatment of teeth with apical periodontitis. Int Endod J 30: 297-30.

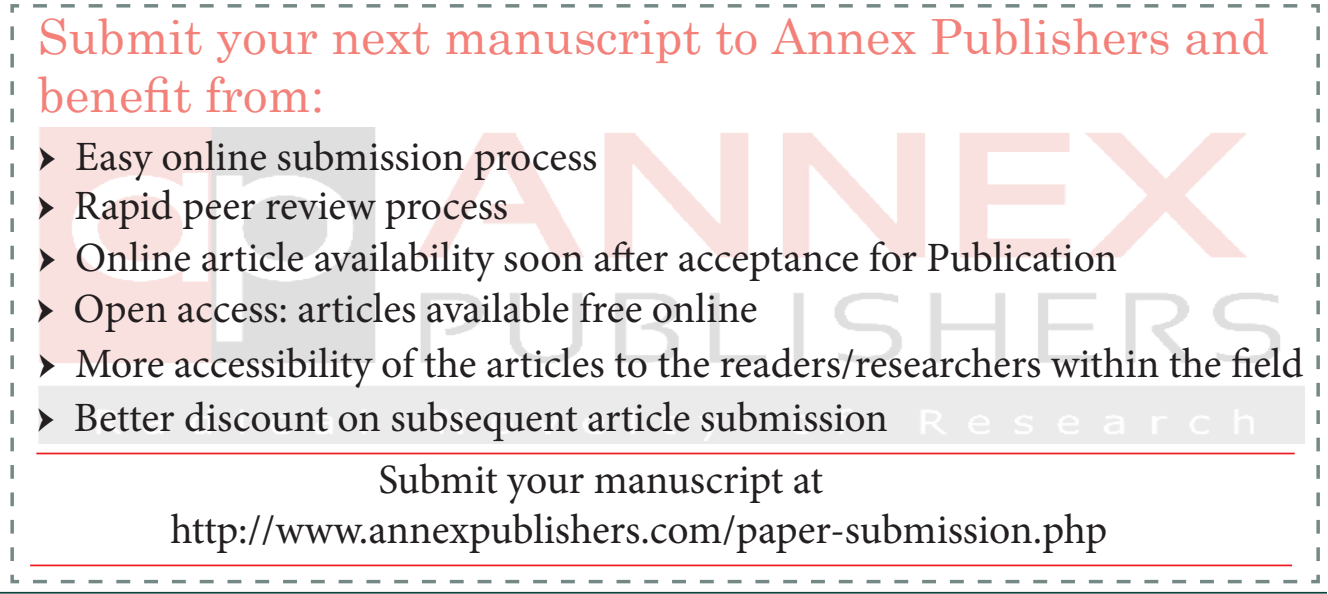

